\title{
Electron Current Measurement of Helical Nonneutral Plasmas for Investigating Plasma Disruption Observed in CHS Experiments
}

\author{
H. HIMURA, Y. YAMAMOTO, A. SANPEI, S. MASAMUNE, M. ISOBE ${ }^{1)}$, S. OKAMURA ${ }^{1)}$ \\ and K. MATSUOKA ${ }^{1)}$ \\ Kyoto Institute of Technology, Department of Electronics, Matsugasaki, Kyoto 606-8585, Japan \\ ${ }^{1)}$ National Institute for Fusion Sciences, Toki, Gifu 509-5292, Japan
}

(Received 4 December 2006 / Accepted 25 March 2007)

\begin{abstract}
A nonneutral plasma confined on helical magnetic surfaces exhibits instability, although it is theoretically stable. In order to investigate the disruptive phenomenon in detail, electron particle flow into a probe tip is carefully measured inside the magnetic surfaces. Preliminary results indicate that the observed disruption is first to occur near the top of the plasma, and then, seems to propagate toward the center of the plasma.
\end{abstract}

(C) 2007 The Japan Society of Plasma Science and Nuclear Fusion Research

Keywords: helical nonneutral plasma, magnetic surface confinement, disruption of nonneutral plasma, electrostatic probe measurement

DOI: $10.1585 /$ pfr.2.S1089

\section{Introduction}

While nonneutral plasmas trapped in purely toroidal magnetic fields have been studied intensively for five decades [1], nonneutral plasmas confined in "toroidal magnetic surfaces" without any electric fields $\mathbf{E}$ have been just investigated [2]. The method of confining electrically nonneutral plasmas in closed magnetic surfaces offers a possibility of re-proportioning electrons and ions inside magnetic surfaces because the magnetic field is completely closed. This property may be applied to produce various fascinating plasmas such as two-fluid plasmas or electronpositron plasmas.

Most studies on the nonneutral plasma confined on magnetic surfaces are so far theoretical works. One of surprising results from them is that unlike the Penning trap in a linear device [3], the energy principle for the plasma has conduced that the plasma stands at its minimum energy state, thus is completely stable [4]. In addition, toroidal magnetic surfaces inherently possess a magnetic shear that would short-circuit such electrostatic modes as the diocotron mode [5] and stabilize them. However, experiments on the Compact Helical System (CHS) machine [6] have shown that the nonneutral plasma confined on magnetic surfaces does not seem to be the case; the plasma exhibits disruption.

The CHS nonneutral plasma confined on "helical magnetic surfaces" lasts about several milliseconds and then disrupts drastically [7]. The duration of the CHS nonneutral plasma is almost same as the electron-neutral collision time so that some ion-related instability [8] has been considered as the most promising mechanism to cause the observed disruption. Surprisingly, similar results are observed in another stellarator device [9], although it is op-

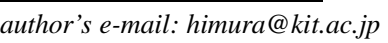

erated under completely different parameter regime from CHS nonneutral plasmas. Thus, this result suggests the existence of a universal mechanism that should not depend on plasma parameters themselves (such as electron density $n_{e}$ ) but others (such as residual gas pressure $P_{0}$, the $E \times B$ speed, and so on). In fact, it is widely known that the ion resonance instability [8] is closely related with $P_{0}$.

In order to examine the detail of the disruption event, we have recently started to measure the time evolution of electron current $I_{e}(z, t)$ with an electrostatic probe [10] on each magnetic surface at a poloidal cross-section. Data are obtained to investigate where the disruption event occurs and how it propagates inside the CHS nonneutral plasmas. In this contributed paper, we present findings obtained from the measured data of $I_{e}(z, t)$ and discuss further things on the observed disruption.

\section{Experimental Setup}

\subsection{Apparatus}

CHS is one of middle-sized stellarator devices for studies on fusion plasmas. The major $R$ and average minor radii $\bar{r}$ are 1.0 and $0.2 \mathrm{~m}$, respectively. A schematic drawing of CHS can be found in Ref. 6. The key parameter which identifies the helical magnetic configuration is the magnetic axis $R_{a x}$ that is usually fixed at $R=101.6 \mathrm{~cm}$. For this case, the stochastic magnetic region (SMR) is present where the last closed flux surface (LCFS) is completely detached from the vacuum chamber [10]. As a result, the helical magnetic surfaces are electrically isolated. The typical magnetic field strength $B$ is $0.9 \mathrm{kG}$ at $R_{a x}$, which yields $\rho_{e} \sim 1.3 \mathrm{~mm}$ when the maximum speed of electrons is $v_{e} \sim 2 \times 10^{7} \mathrm{~m} / \mathrm{s}$ for $e V_{a c c} \sim 1.2 \mathrm{keV}$, where $\rho_{e}, v_{e}$, and $e V_{a c c}$ are the Larmor radius, the electron velocity, and the 
beam energy, respectively.

On the CHS machine, we have studied on helical nonneutral (pure electron) plasmas having the electron temperature $T_{e}$ up to $\sim 250 \mathrm{eV}$. Electrons are injected into SMR from a diode-type electron gun which equips a $\mathrm{LaB}_{6}$ emitter as the cathode. Values of $e V_{a c c}$ are changeable, but in presented experiments it is fixed to the value which varies in the range between $-250 \mathrm{~V}$ and $-1.2 \mathrm{kV}$.

\subsection{Probing}

Because of charge nonneutral, the space potential $\phi_{s}$ of nonneutral plasmas can become very large. For CHS nonneutral experiments, the value of $\phi_{s}$ depends on $e V_{a c c}$ [7]. This is because $e V_{a c c}$ is equal to the initial total energy of injected each electron. As mentioned above, the maximum value of $e V_{a c c}$ has been $-1.2 \mathrm{kV}$. Thus, $\phi_{s}$ reaches, in principle, up to $-1.2 \mathrm{kV}$ as well. Obviously, this potential well is deeper (negatively larger) than that of conventional neutral plasmas. Nevertheless, the value of $n_{e}$ is relatively small, which is in typical about $10^{11-13} \mathrm{~m}^{-3}$ for CHS nonneutral experiments. These properties, therefore, require somewhat modifications even to a simple electrostatic probe when being applied to CHS nonneutral plasmas. The detail of the modified technique for the probe is explained in a companion paper [10]. Using the probing, both $\phi_{s}$ and $I_{e}$ have been measured in CHS nonneutral experiments [11].

\section{Observed Disruption of Nonneutral Plasmas Confined on CHS Mag- netic Surfaces}

As mentioned in Sec. 1, the nonneutral plasma confined on helical magnetic surfaces turns to be unstable during its equilibrium state. This event is always observed in CHS nonneutral experiments. Figure 1 (a) shows a typical time evolution of $\bar{I}_{e}\left(\equiv I_{e} / e S\right)$ measured in the helical magnetic surfaces. Here, $e$ and $S$ are the electron charge and the surface area of the probe tip. Electrons are injected at $t \sim 0.5 \mathrm{~ms}$, and $\bar{I}_{e}$ immediately increases up within $\sim$ $100 \mu$ s to its saturation value $\left(\sim 8 \times 10^{20} / \mathrm{m}^{2} \mathrm{~s}\right)$. However, at $t \sim 1.7 \mathrm{~ms}$, it is recognized that $\bar{I}_{e}$ decreases drastically, indicating that some disruption occurs. At this moment, the value of $\bar{I}_{e}$ once decreases down to $\sim-1 \times 10^{21} / \mathrm{m}^{2} \mathrm{~s}$, and then, shoots up again quickly. Subsequently, this event is repeated. This is because in experiments electrons are continuously injected so that such multiple disruptions are occurred. Regarding the growth rate of the disruption, it is about $50 \mu$ s (see Fig. 1 (b)) and its frequency is $\sim 100 \mathrm{kHz}$. Similar significant drop has been recognized also in measured $\phi_{s}(t)$.

Actually, the above disruption seems to limit the life time of CHS nonneutral plasmas. For example, the particle confinement time $\tau_{N}$ can be estimated from the time history of $\bar{I}_{e}(t)$ measured at the LCFS, that is, the plasma edge. Data have shown that $\bar{I}_{e}(t)$ persists for $\sim 1.5 \mathrm{~ms}$ after

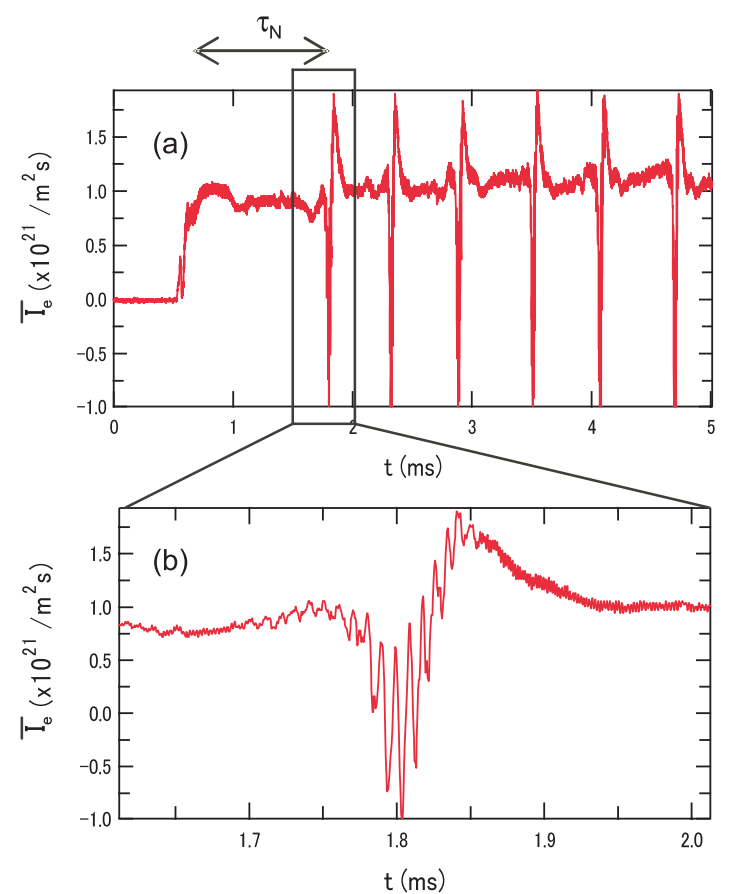

Fig. 1 (a) A typical time evolution of normalized probe currents $\bar{I}_{e}\left(\equiv I_{e} / e S\right)$ of the CHS nonneutral (pure electron) plasmas. Data shows an event of multiple disruptions of the plasma. Thus, the confinement time $\tau_{N}$ of the plasma is inferred to be $\sim 1.5 \mathrm{~ms}$. (b) An enlarged figure of the first disruption occurred at $t \sim 1.8 \mathrm{~ms}$, as seen in (a).

the electron injection is turned off, thus indicating that $\tau_{N}$ is $\sim 1.5 \mathrm{~ms}$.

\section{The Measured $I_{e}\left(\Psi^{1 / 2}, t\right)$ Inside the LCFS}

At the last series of experiments on CHS, we have measured $\bar{I}_{e}(t)$ on each magnetic surface by carefully moving the probe. A set of the whole data is described in Fig. 2, which shows the evolution of $\left.\bar{I}_{e} \Psi^{1 / 2}, t\right)$ at the poloidal cross-section where the probe is installed. Remarkably, the result reveals several notable points that have never been noticed in past experiments. First, at $t \sim 4 \mathrm{~ms}$, the onset of the disruption is observed at $\Psi^{1 / 2} \sim 0.7$, where $\Psi^{1 / 2}$ is the averaged minor radii; one notes that $\Psi^{1 / 2}=0$ and 1 correspond to $R_{a x}$ and LCFS, respectively. As recognized, $\bar{I}_{e}$ at all measurement points rise up immediately just after electrons are injected at $t \sim 3 \mathrm{~ms}$. And then, at $t \sim 4 \mathrm{~ms}$, $\bar{I}_{e}$ at $\Psi^{1 / 2} \sim 0.7$ is first to start up fluctuating, which indicates that the disruption happens. It is worth noting that other than the place $\left(\Psi^{1 / 2} \sim 0.7\right)$, no perturbed signal can be seen at the onset time $(t \sim 4 \mathrm{~ms})$. Therefore, this result implies that the observed disruption is one of branches of local modes, not global modes. Second, interestingly, the disruption seems to start only at the upper side $(z>0)$ from the equatorial plane (located at $z=0$ ) of the CHS machine. In fact, looking at the data of $\bar{I}_{e}$ measured at $\Psi^{1 / 2} \sim-0.7$, 


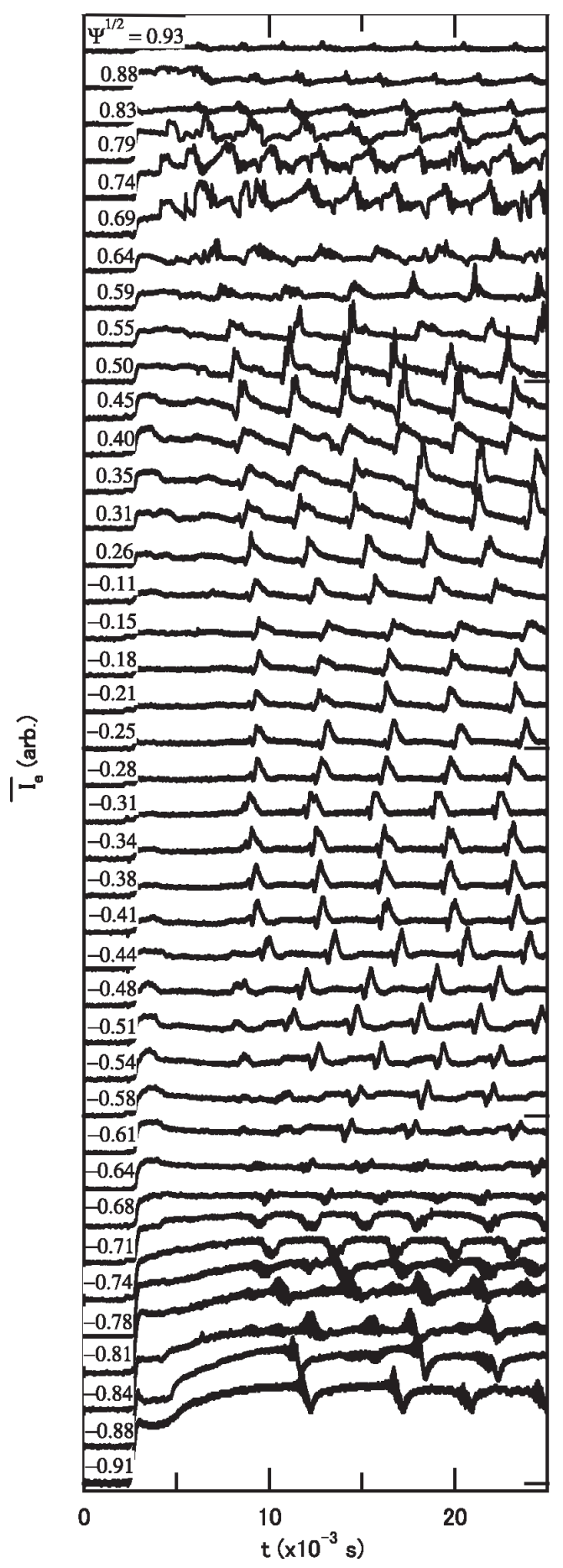

Fig. 2 Time evolutions of $\bar{I}_{e}$ in CHS helical magnetic surfaces for $R_{a x}=101.6 \mathrm{~cm}$ and $B=0.9 \mathrm{kG}$. The numbers of $\Psi^{1 / 2}$ represent values of averaged minor radii.

no apparent change can be recognized at $t \sim 4 \mathrm{~ms}$ when the first sign of the disruption certainly appears at the upper side $(z>0)$ on the same magnetic surface.

It is considered that no significant difference between the upper- and the lower-side on the same magnetic surface would exist in the poloidal cross-section. On the other hand, there is considerable difference in both $\phi_{s}$ and $n_{e}$.

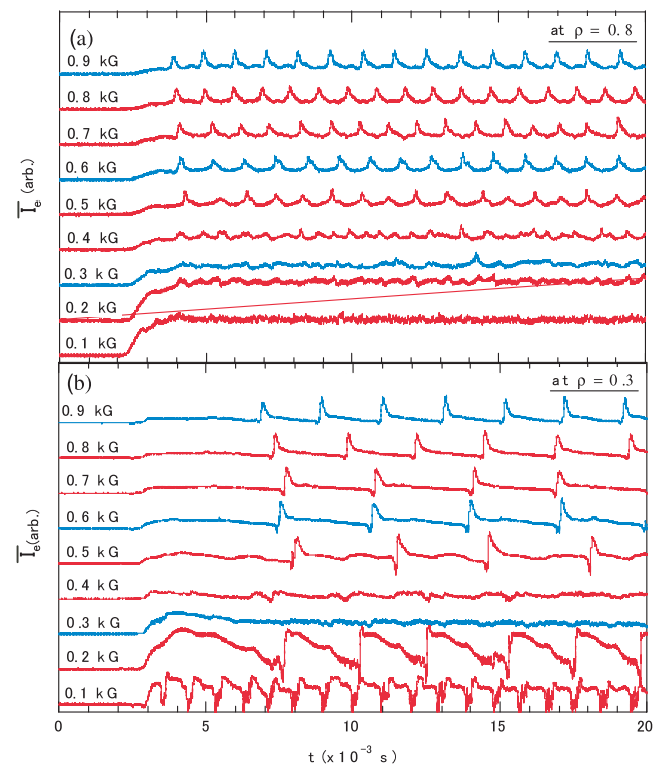

Fig. 3 (a) Time evolutions of $\bar{I}_{e}$ measured with different $B$ strength. Data are measured near the plasma edge (at $\left.\Psi^{1 / 2} \sim 0.8\right)$. (b) Time evolutions of $\bar{I}_{e}$ measured near the core of the plasma (at $\Psi^{1 / 2} \sim 0.3$ ).

Recently it is confirmed in experiments that those are "non-constant" parameters on each helical magnetic surface when the plasma is in its equilibrium [12]. Thus, this result obviously would bring in inhomogeneous structures of $\mathbf{E}$ and $\mathrm{d} \mathbf{E} / \mathrm{d} \boldsymbol{r}$ in the magnetic surfaces. As a result, some condition for triggering the observed disruption may be satisfied only at $\Psi^{1 / 2} \sim 0.7$.

Thirdly, the perturbed signal of $\bar{I}_{e}$ generated at $\Psi^{1 / 2} \sim$ 0.7 seems to propagate towards the center of magnetic surfaces with very slow speed, which is the order of $10^{2} \mathrm{~m} / \mathrm{s}$. Moreover, at most measurement points, when being perturbed, values of $\bar{I}_{e}$ increase (like a "bump"). In fact, this could be happened as a consequence of the observed disruption. This is because $\phi_{s}$ has decreased when the disruption occurs (see also Fig. 1), which thus causes the potential well to be shallower. Consequently, electrons emitted newly from the $\mathrm{LaB}_{6}$ cathode would immediately try to recover its equilibrium value of $\phi_{s}$ (deeper negative potential), thus tending to move to the plasma core.

Similar experiments were performed with different $B$ strength. Figure 3 (a) shows a reconstruction data based on $\bar{I}_{e}(t)$ in magnetic surfaces. Data are measured near the plasma edge (at $\Psi^{1 / 2} \sim 0.8$ ). In experiments, the strength of $B$ has been changed from 0.1 to $0.9 \mathrm{kG}$. As is the case with Fig. 2, the disruption occurs when $B$ is reduced. However, the time interval between the multiple disruptions seems to become longer. We will come back to this point later.

Remarkably, the sign of disruption appears more clearly with stronger $B$ strength, not weaker one. In fact, when $B$ is less than $0.3 \mathrm{kG}$, the spike signal observed in 


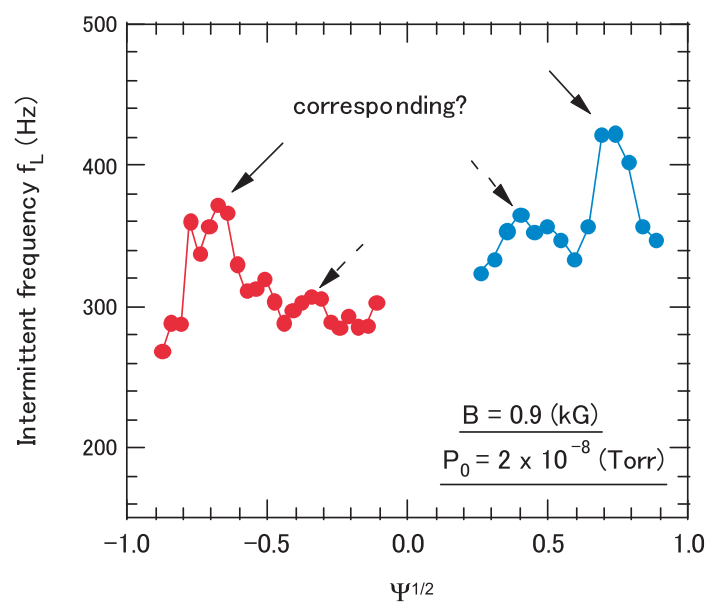

Fig. 4 The intermittent frequency $f_{L}$ plotted against $\Psi^{1 / 2}$. Here, $f_{L}$ is defined as the time interval between successive disruptions.

$\bar{I}_{e}(t)$ has almost disappeared. Such tendency, however, is not observed near the core of the plasma (at $\Psi^{1 / 2} \sim 0.3$ ), as recognized from Fig. 3 (b). At this measurement point, the disturbed signal is still clearly seen even when $B \sim 0.1 \mathrm{kG}$. However, careful consideration is called for. Despite the $B$ strength, electrons are strongly magnetized due to the large number of electron cyclotron frequency. Meanwhile, electron Larmor radius is about $1 \mathrm{~cm}$, which is hardly small compared to the length scale of magnetic surfaces. This means that magnetic surface confinement may be dissatisfied; plasma equilibria may thus be broken when $B$ is weaker.

Therefore, only we can conclude from the comparison Fig. 3 (a) with (b) is that the time interval between multiple disruptions (thereafter, called "intermittent frequency") is shorter near the plasma edge than the plasma core. Figure 4 shows the preliminary result of the intermittent frequency plotted against $\bar{r}$. In fact, it varies in the range between $\sim$ 250 and $\sim 420 \mathrm{~Hz}$ in the magnetic surfaces. This result may be other evidence, supporting that the observed disruption is a local mode and driven from the region near the plasma edge.

\section{Summary}

Experimental studies on nonneutral plasmas confined on helical magnetic surfaces have been performed on CHS. Contrary to theoretical prediction, the helical nonneutral plasmas exhibit instability. In order to investigate it in detail, electron currents $I_{e}$ are measured by precisely moving an electrostatic probe and then arranged in the $z$ coordinate order to see the evolution of $I_{e} \Psi^{1 / 2}, t$ ) to examine where the instability is triggered from and how the disturbed signal propagates inside helical magnetic surfaces. A preliminary analysis shows that the disruption occurs at first near the plasma top (at $\Psi^{1 / 2} \sim 0.7$ ) and seems to propagate towards the plasma core. The propagation speed is the order of $10^{2} \mathrm{~m} / \mathrm{s}$, which is very slow compared to all other typical speeds of CHS nonneutral plasmas. Also, the disruption happens only in the upper side $(z>0)$ from the equatorial plane. This result strongly suggests that the observed disruption is some local mode and driven from the region near the plasma edge. In fact, although the detail is still under investigated, the observed disruption may be related with the experimental finding of the non-uniformity of both $n_{e}$ and $\phi_{s}$ on helical magnetic surfaces when the plasma is in equilibrium.

[1] for example, J.D. Daugherty, J.E. Eninger and G.S. Janes, Phys. Fluids 12, 2677 (1969).

[2] C. Nakashima, Z. Yoshida, H. Himura et al., Phys. Rev. E 65, 036409 (2002); T.S. Pedersen, in Stellarator news 99, 3 (2005).

[3] R.C. Davidson, Physics of Nonneutral Plasmas (AddisonWesley Publishing Company, Redwood, CA, 1990) p. 39.

[4] A.H. Boozer, Phys. Plasmas 11, 4709 (2004).

[5] K.S. Fine, C.F. Driscoll and J.H. Malmberg, Phys. Rev. Lett. 63, 2232 (1989).

[6] K. Nishimura, K. Matsuoka, M. Fujiwara et al., Fusion Technol. 17, 86 (1990).

[7] H. Himura et al., will be appeared in the TCP'06 conference proceedings (2007).

[8] R.H. Levy and J.D. Daugherty, Phys. Fluids 12, 2616 (1969).

[9] J.P. Kremer et al., Phys. Rev. Lett. 97, 095003 (2006).

[10] Y. Yamamoto et al., in this conference proceedings (2007).

[11] H. Himura et al., Phys. Plasmas 11, 492 (2004).

[12] H. Himura et al., Phys. Plasmas 14, 022507 (2007). 